\title{
ON THE SPECTRA OF R SCUTI AND W CYGNI
}

Observations of the spectra of R Scuti and W Cygni are particularly valuable, as these stars occupy a unique position between the short-period variables on the one hand and the $o$ Ceti variables on the other. Visual observations of $\mathrm{R}$ Scuti by Espin have led him to suspect the presence of bright lines in its spectrum, but he seems to have been unable to identify them. As far as I know, W Cygni had not been observed with the spectroscope.

Exposures upon both these stars with Spectrograph I were begun in the middle of July, 1903. They were continued until November 11, 1903, in case of R Scuti, and December 28, 1903, in case of W Cygni. R Scuti was examined visually with the spectrograph until December 7. In the meantime, $\mathrm{R}$ Scuti rose to maxima toward the end of July and the beginning of October, while maxima of W Cygni occurred early in August and in the middle of December. During this period about 25 spectrograms of $\mathrm{R}$ Scuti and 20 of W Cygni were secured with Spectrograph I.

On plate $13 \mathrm{E}$ of R Scuti (see Plate 4), $\mathrm{H} \beta, \mathrm{H} \gamma$, and $\mathrm{H} \delta$ shone out strongly as bright lines, but they faded quickly until at minimum the spectrum of the star departed but little from the solar type, with dark hydrogen lines of customary intensity. At the two subsequent maxima the bright hydrogen lines were not seen, but the intensity of the absorption line at $\mathrm{H} \gamma$ seemed to have decreased very much as the star approached its maximum of October (See Plate 5). Judging from rough measures of a few plates, the radial velocity of this star appears to be constant and about $+42 \mathrm{~km}$. in actual value.

W Cygni shows a banded spectrum of a type characteristic of long-period variables. At the maximum of August, 1903, the hydrogen lines mentioned above appeared as strong bright lines (see Plate 6) which faded gradually to the star's minimum (see Plate 7 ), while the absorption line at $g$ broadened greatly during the same period. Other suspected bright lines in the spectrum this star remain to be identified.

A detailed study of these plates will be made at the earliest opportunity.

RalPh H. Curtiss. 